\title{
Effect of Bletilla striata on the Prevention of Postoperative Peritoneal Adhesions in Abrasion-Induced Rat Model
}

\author{
BoTong Liu $\mathbb{D},{ }^{1}$ QiuHua Zhang $\mathbb{D},{ }^{1}$ Xiao Wu $\mathbb{D},{ }^{2}$ YanJun Fu $\mathbb{D},{ }^{1}$ Hui Wang $\mathbb{D},{ }^{1}$ \\ YunXia Guan $\mathbb{D}$, ${ }^{1}$ ShiCheng Yang $\mathbb{D},{ }^{1}$ YiLan Liu, ${ }^{1}$ WenCong Cao $\mathbb{D}^{1},{ }^{1}$ and Jian Wang $\mathbb{D}^{3}$ \\ ${ }^{1}$ Department of Basic Pharmacology, Liaoning University of Traditional Chinese Medicine, 110032 Shenyang, China \\ ${ }^{2}$ Department of Animal Experimental Center, HE's University, 110163 Shenyang, China \\ ${ }^{3}$ Department of Laboratory Animal Center, Liaoning University of Traditional Chinese Medicine, 110032 Shenyang, China
}

Correspondence should be addressed to QiuHua Zhang; 1051374105@qq.com

Received 31 January 2019; Revised 21 April 2019; Accepted 13 May 2019; Published 10 June 2019

Academic Editor: Caigan Du

Copyright (C) 2019 BoTong Liu et al. This is an open access article distributed under the Creative Commons Attribution License, which permits unrestricted use, distribution, and reproduction in any medium, provided the original work is properly cited.

Postoperative peritoneal adhesions (PPAs) constitute a common complication of abdominal surgery with a high incidence. Bletilla striata (BS) is an important hemostatic drug used in China for nearly 2000 years. The purpose of this study was to investigate the effect of Bletilla striata on postoperative intestinal adhesion in rats. PPA was induced by cecal wall abrasion, and Bletilla striata was injected to observe its effect on adhesion in rats. The adhesion and inflammation score were assessed through visual observation and histopathologic evaluation. The levels of interleukin-1 (IL-1 $\beta$ ), tumor necrosis factor (TNF- $\alpha$ ), and interleukin-17F (IL-17F) in abdominal cavity and interleukin-6 (IL-6) in plasma were measured by enzyme-linked immunosorbent assay (ELISA) at 6 hours, 12 hours, 24 hours, and 1 week after operation. The tissue level of transforming growth factor beta-1 (TGF- $\beta 1$ ) was also determined by ELISA on the seventh day after surgery. The expressions of collagen and TNF- $\alpha$ were, respectively, detected by Masson trichrome staining and immunohistochemical staining. The expression of TGF- $\beta 1$ and alpha smooth muscle actin ( $\alpha$-SMA) was detected by Western blot. The result showed that Bletilla striata has obvious preventive effect on PPAs and celiac inflammation of PPAs. Bletilla striata could significantly reduce the level of IL-17F abdominal cavity and IL-6 in plasma. Masson trichrome staining and immunohistochemical staining results showed that Bletilla striata also decreased the expression of TNF- $\alpha$ and collagen. Western blot results showed that Bletilla striata decreased the expression of $\alpha$-SMA and TGF- $\beta 1$. Our results suggest that Bletilla striata decreased the development of abdominal adhesion in abrasion-induced model of rats and reduced the expression of the important substance which increased in PPAs. Bletilla striata can be further studied as a new and cheaper antiadhesive substance.

\section{Introduction}

Postoperative peritoneal adhesions (PPAs) constitute a common complication of abdominal surgery; the incidence rate is up to $90 \%-95 \%$ [1]. PPAs may cause significant morbidity, including adhesive small bowel obstruction, female infertility, chronic abdominal pain, and increased difficulty with subsequent surgery $[2,3]$. The formation of PPAs involves a complex interaction of cytokines, growth factors, cell adhesion molecules, neuropeptides, and numerous other factors secreted by cells in or near the area of trauma $[4,5]$. Preventive measures include keeping the lesions separated, increasing bowel motility, and decreasing local inflammation [6]. Currently, there is no widely accepted method of treating abdominal adhesions, so we still need to find a more effective treatment method to prevent abdominal adhesion after operation.

Bletilla striata (Thunb.) Reichb.f. (Orchidaceae) is an important astringent hemostatic medicinal plant used for almost 2000 years in China and is widely distributed in eastern and southern Asia. Many studies have demonstrated that it exhibits wound healing activity, antiulcer activity, hemostatic activity, and anti-inflammatory activity [7]. Recently, a research confirmed its protective effect on renal fibrosis, which is probably mediated by downregulated alpha smooth muscle actin ( $\alpha$-SMA) in vitro. [8]; therefore, we speculate that Bletilla striata can prevent abdominal adhesion after operation. 
Although the mechanism of abdominal adhesion after operation is not very clear, it represents exaggerated and dysregulated peritoneal "repair" mechanisms in response to trauma, leading to a series of local responses involving acute inflammation, extracellular matrix (ECM) deposition, fibrinolysis, and neoangiogenesis $[9,10]$. Therefore, the local inflammation and transforming growth factor (TGF)- $\beta$ family are the important factors of abdominal adhesion after operation. Hence, the main purpose of this study was to investigate the effect of Bletilla striata on abdominal adhesions, inflammatory factors and related fibrogenic factors such as TGF- $\beta 1$ in the cecum tissue to verify our hypothesis that the inhibitory effect of Bletilla striata on PPAs is closely related to the inhibition of TGF- $\beta 1$ and Bletilla striata can be further studied to develop into a therapeutic drug for the prevention of abdominal adhesions.

\section{Materials and Methods}

2.1. Chemicals and Biochemicals. The ELISA Kits of IL$1 \beta$ (EK0393), IL-6 (EK0412), TNF- $\alpha$ (EK0526), and IL$17 \mathrm{~F}$ (EK1136) were purchased from the Boster Biological Co., Ltd. The ELISA Kit of TGF- $\beta 1$ (m10021856) was purchased from Mlbio Biological Co., Ltd. HRP AffiniPure Goat Anti-Rabbit IgG (E030120) and GAPDH (E021060-03) were from EarthOx Life Sciences. SDS-PAGE loading buffer (5X) (P0015), Western and IP lysis buffer (P0013), ECL luminous solution (P0018), Primary and Secondary Antibody Dilution Buffer (P0023A, P0023D), BCA Kit (P0012S), and SDS-PAGE Gel Kit (P0012A) were purchased from Beyotime Biological Co., Ltd. $\alpha$-SMA monoclonal antibody (\#19245) and TNF$\alpha$ polyclonal antibody (WL01896) were, respectively, from Cell Signaling and Wanleibio Limited Companies. Protease Inhibitor Cocktail (A8260) and Histostain-SP Kits (SPN9001) came from Solarbio Science and Technology Co., Ltd. and Zsbio Commerce Store.

2.2. Extraction of Plant Material. Bletilla striata was purchased from Department of Pharmacy, Affiliated Hospital of Liaoning University of Traditional Chinese Medicine, and was identified as the original plant of Bletilla striata (Thunb.) Rchb.f. by the professor Wang Weining, Liaoning Institute of Pharmacology. An amount of $150 \mathrm{~g}$ Bletilla striata was collected for drying, crushing, and cold soaking before aqueous extraction; then $1000 \mathrm{ml}$ distilled water was added to boil for $45 \mathrm{~min}$. After collecting the filtrate, the residue was boiled twice for $30 \mathrm{~min}$ with $500 \mathrm{ml}$ distilled water. Subsequently, three times of filtration solution evaporated and condensed into $50 \mathrm{ml}$ decoction, then the decoction was added to ethanol, refrigerated, filtered, and recovered. The decoction was added to injection water and $2 \%$ activated carbon to $1000 \mathrm{ml}$ and boiled for $30 \mathrm{~min}$, filtered and packed in a bottle, and sterilized for further usage.

2.3. Animals and Diets. 80 male Sprague-Dawley rats (Liaoning Changsheng Biotechnology co., Ltd.) weighing 169 $206 \mathrm{~g}$ were acclimated for one week before the experiments. They were housed in controlled room (temperature: $20-25^{\circ} \mathrm{C}$, relative humidity: $40 \%-70 \%$, air circulating frequency: 20 times/hour, artificial light: 15-20Lux from 8:00 AM to 8:00 PM, noise: $<60 \mathrm{db}$ ). Rats were given standard rat chow and free access to water. This experiment was approved by the ethical test of animal experiment of Liaoning University of Traditional Chinese Medicine (No. 21000092017048).

2.4. Study Design. The adhesion model in this study is based on the adhesion model described by Hemadeh et al. [11]; the method can cause $100 \%$ abdominal adhesion in rats 7 days after abdominal operation. Rats were only provided with water before the operation and weighed and anesthetized by intramuscular injection of chloralic hydras, breathing spontaneously throughout the procedures. The abdominal skin of rats was shaved and cleaned with povidone-iodine solution. The operation was performed under aseptic conditions. In each rat, a $3 \mathrm{~cm}$ vertical midline incision was made, and then the abdomen was opened. Except for the sham operation group, the caecum of rat in the three groups was exposed to the ventral and dorsal surfaces and gently rubbed with 2 dry gauze pieces until the gloss and bleeding point appeared, and the caecum was recovered to the anatomic position. Sham operation group was subjected to midline incision of the abdomen without any scratch. After abdominal closure, animals were randomly divided into 4 groups with 32 animals in each group. The rats in the sham operation group was not treated; the other rats of the three experimental groups were intraperitoneally injected with $8 \mathrm{ml}$ of phosphate-buffered saline (Control group), 15\% Bletilla striata extraction solution (BS group), and $0.2 \%$ hyaluronic acid solution (HA group), respectively. The concentration of HA was similar to that of Reijnen et al. [12] as a positive control group. The dose of Bletilla striata is lower than the minimum dose of human-rat conversion as stipulated in the Chinese Pharmacopoeia 2015, which means that the dose of Bletilla striata used for rats is the safe dose.

All animals were given water only on the first day and given normal diet and water on the second day after operation. A week later, all rats were euthanized and the abdomens of the rats were opened through a U-shaped incision, thoroughly explored.

2.5. Adhesion Grading and Evaluation. Methods described by Nair et al. [13] were used to determine the degree of adhesion on the 7 days after operation. This scoring system is based on the following criteria: 0 represents the presence of no adhesive bands; 1 represents a single adhesive zone between the viscera or between the viscera and the abdominal wall; 2 represents two bands formed between the viscera or between the viscera and the abdominal wall; 3 represents more than two bands formed between the viscera or between the viscera and the abdominal wall, or the whole intestine formed a mass without attachment to the abdominal wall; 4 indicates that the viscera are directly attached to the abdominal wall without being affected by the number of bands.

2.6. Sample Collection. Blood was collected through abdominal aorta by vacuum tube (with heparin). Peritoneal fluid 
was perfused with $10 \mathrm{ml}$ PBS. Samples were transferred to centrifuge at $4^{\circ} \mathrm{C}(120 \times \mathrm{g})$ for $3 \mathrm{~min}$ and stored at $-80^{\circ} \mathrm{C}$ until analysis. The adhesive caecum was carefully removed, and the tissue was cut longitudinally to remove contents and washed with aseptic PBS. Half of the tissues in each group were fixed in $10 \%$ formalin in PBS for histopathologic evaluation, immunohistochemistry, and Masson trichrome staining. The other half of tissues in each group were stored at $-80^{\circ} \mathrm{C}$ for ELISA and Western blot analysis.

2.7. Histopathologic Evaluation. The cecal was fixed in $10 \%$ formalin in PBS for at least 1 hour. After routine processing, serial sections were stained with hematoxylin and eosin. The other sections were stained with Masson trichrome stain. The degree of inflammation was evaluated in a double-blind study by two assessors using a scoring system described by GD et al. [14]: grade 1, macrophages, occasionally scattered around lymphocytes and plasma cells, indicate a mild inflammatory reaction; grade 2 , macrophages increased and admixed with lymphocytes, plasma cells, eosinophils, and neutrophils, mean a moderate reaction; grade 3 , the presence of microabscesses means a severe inflammatory response.

2.8. Enzyme-Linked Immunosorbent Assays (ELISA). The concentrations of IL- $\beta 1$, IL-6, TNF- $\alpha$, and IL-17F in peritoneal fluid and IL-6 in plasma were determined by ELISA kit. The concentration of TGF- $\beta 1$ in cecum tissue was detected according to the manufactures' instructions.

2.9. Immunohistochemistry. Immunohistochemical staining was performed by using a Histostain-SP Kits following the manufacturer's instructions. The sections were deparaffinized and rehydrated, then incubated with $30 \mathrm{~g} / \mathrm{L} 3 \%$ hydrogen peroxide solution at room temperature for 10 minutes, and washed 5 times in distilled water. After antigen repair, the sections were incubated in blocking buffer. These sections were incubated overnight at $4^{\circ} \mathrm{C}$ with antibody TNF- $\alpha$. After the section was washed repeatedly, biotinylated secondary antibody working solution and horseradish peroxidaseconjugated streptavidin working solution were added, respectively. The slices were washed in PBS ( $\mathrm{pH} 7.2)$ for 5 minutes. Diaminobenzidine tetrahydrochloride (DAB) was used for visualization, and hematoxylin was used as the counterstain; the sections were dehydrated, mounted, and sealed. An image signal acquisition and analysis system (Leica) was used for image acquisition. Positive cells were counted as a brown deposit in cells, made in randomly selected high power fields $\left(400 \times ; 2.37 \mathrm{~mm}^{2}\right)$ [15].

2.10. Western Blotting. Western blotting was prepared according to the procedure used by Guo et al. [16]. The cecum or peritoneum of rats was placed in a lysis buffer containing $10 \mathrm{u}$ L phosphatase inhibitor, $1 \mathrm{u}$ L protease inhibitor, and $5 \mathrm{u}$ L 100 mM PMSF. After boiling for $5 \mathrm{~min}, 75$ micrograms of protein was dissolved in the loading buffer and denatured. Each sample was subjected to electrophoresis on $10 \%$ SDS polyacrylamide gel. Then the protein was transferred to the PVDF membrane. After blocking the membrane for $1 \mathrm{~h}$ at room temperature with blocking buffer, the membranes were probed with primary antibodies including TGF- $\beta 1$ (1/1000 dilution), $\alpha$-SMA (1/1000 dilution), and GAPDH (1/5000 dilution) as appropriate with constant shaking overnight at $4^{\circ} \mathrm{C}$. After washing with $0.1 \%$ Tween-20 in TBS for three times, the secondary antibody was incubated in the buffer at room temperature for $1 \mathrm{~h}$. The bound antibodies were detected with enhanced chemiluminescence system (Millipore) as recommended by the manufacturer.

2.11. Bioinformatics. We used TCMID, the world's largest database of traditional Chinese medicine ingredients, to search for the active ingredients of Bletilla striata [17]. Then we used BATMAN-TCM to analyze the biopathway enrichment of the active ingredients of Bletilla striata in the Kyoto Encyclopedia of Genes and Genomes (KEGG) [18]. The significantly enriched paths of the active ingredients of Bletilla striata were chosen to draw the bar chart $(\mathrm{P}<0.05)$.

2.12. Statistical Analysis. Quantitative data were expressed as the mean \pm SEM. The Kruskal-Wallis test was used to observe the degree of the adhesion and inflammatory degrees. Oneway ANOVA analysis was used to evaluate the differences among the four groups. Fisher's least significant difference (LSD) test was used to determine the differences between each group. The significance level was set at $\mathrm{P}<0.05$. Statistical analysis was performed using SPSS 19.0 software.

\section{Results}

3.1. Bletilla striata Reduced Abdominal Adhesion Scores in Rats. 128 male Sprague-Dawley rats completed the whole experiment, and no death occurred among the four groups of rats. Figure 1(a) is a typical image of abdominal adhesion in rats of each group. Based on the Nair's scoring system, the average adhesion score per group is shown in Figure 1(b). The adhesion score of the model group was significantly higher than that of the sham operation group $(\mathrm{P}<0.001)$, which proved that the model was made successfully. The adhesion score of Bletilla striata group and HA group was significantly lower than that of model group, indicating that Bletilla striata and HA could inhibit PPAs.

3.2. Bletilla striata Alleviates the Inflammatory Reaction of PPAs in Rats. In our study, we observed that the inflammatory reaction of PPAs mainly occurred in the mucosa, submucous membrane, and muscle layer by the abdominal adhesion, H.E staining, and immunohistochemical staining. The control group showed increased admixed lymphocytes, plasma cells, eosinophils, and neutrophils (inflammation grade 3) (Figure 2(a)). Compared with the control group, the hyperemia and inflammatory reaction of rats treated with Bletilla striata and HA were obviously alleviated (Figure 2(b)); the scores of inflammatory response in BS group and HA group were significantly lower than those in control group (Figure 2(b)); the results showed that Bletilla striata alleviates the inflammatory reaction of PPAs in rats. 


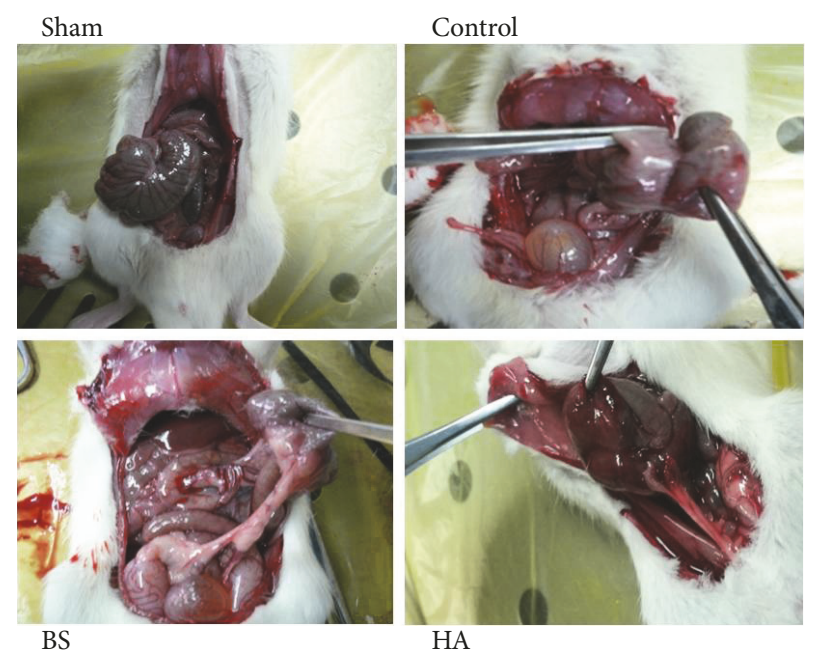

(a)

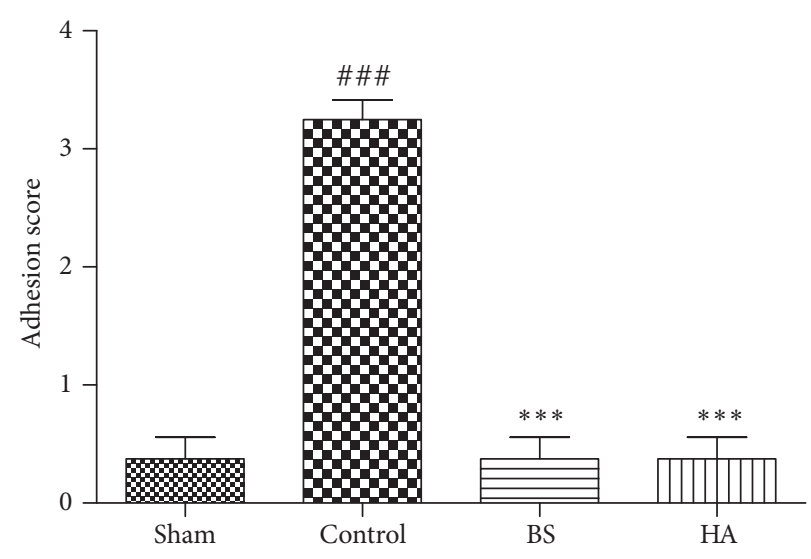

(b)

FIGURE 1: Bletilla striata reduced postoperative intestinal adhesion in rats. (a) The typical image of abdominal adhesion in each group rats. Sham operation group: no adhesions; control group: the whole cecum forms a mass without adhering to the abdominal wall; BS and HA group: a single adhesion band formed between the viscera or between a viscus and the abdominal wall. (b) Adhesion scores in each group, $\# \# \mathrm{P}<0.001$ compared with sham laparotomy group. $* * * \mathrm{P}<0.001$ compared with control group.

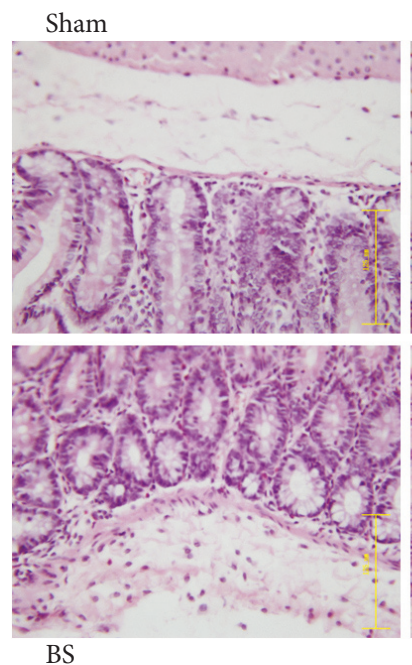

(a)
Control

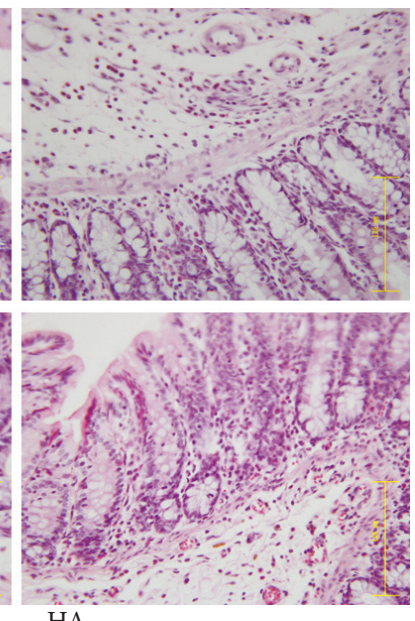

HA

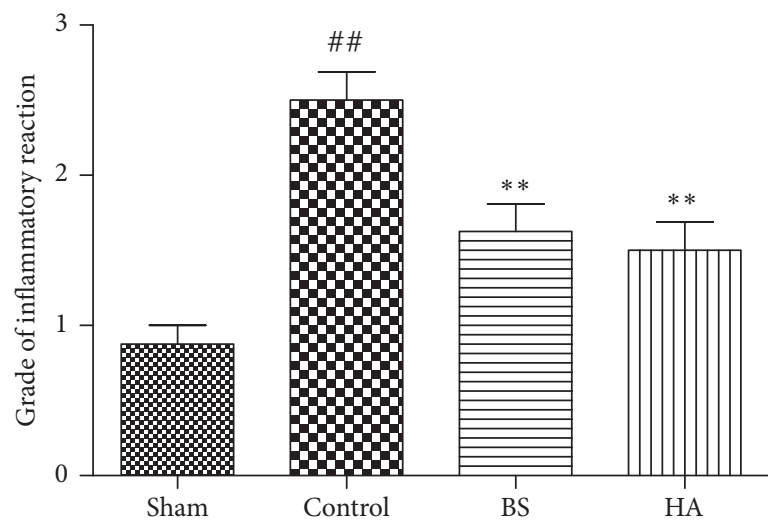

(b)

FIGURE 2: Bletilla striata alleviates the inflammatory reaction of postoperative intestinal adhesion in rats. (a) The typical image of inflammatory response among sham laparotomy, control, BS, and HA group by H.E. $(400 \times)$. (b) The grade of inflammatory reaction in each group $(\mathrm{n}=8)$. $* * \mathrm{P}<0.01$ compared with the control group. $\# \# \mathrm{P}<0.01$ compared with the sham group.

\subsection{Bletilla striata Reduces the TNF- $\alpha$ Expression in Caecum} on the 7th Day after Operation. TNF- $\alpha$ immunohistochemical staining in abdominal adhesion tissue showed that the cell numbers of TNF- $\alpha$ expressing in control group were significantly higher than that in the sham operation group. The expression of TNF- $\alpha$ in caecum of rats treated with Bletilla striata and HA was significantly lower than that of control group $(\mathrm{P}<0.01)$ (Figure 3 ); the results showed that Bletilla striata inhibited the TNF- $\alpha$ expression in caecum on the 7 th day after operation.
3.4. Bletilla striata Inhibited the Levels of IL-6 in Plasma and IL-17F in Peritoneal Fluid after PPAs in Rats. The levels of IL6 in plasma and IL- $\beta 1$, TNF- $\alpha$, and IL-17F levels in peritoneal fluid were measured by ELISA assay at 6 h, 12 h, and 1 week after operation. The results showed that the levels of IL-6 in plasma and IL-17F in peritoneal fluid in the control group were significantly higher than those in the sham operation group at $6 \mathrm{~h}$. Compared with the control group, both Bletilla striata and HA inhibited the secretion of IL- 6 in plasma and IL-17F in peritoneal fluid $(\mathrm{P}<0.01)$ (Figure 4$)$. There was 


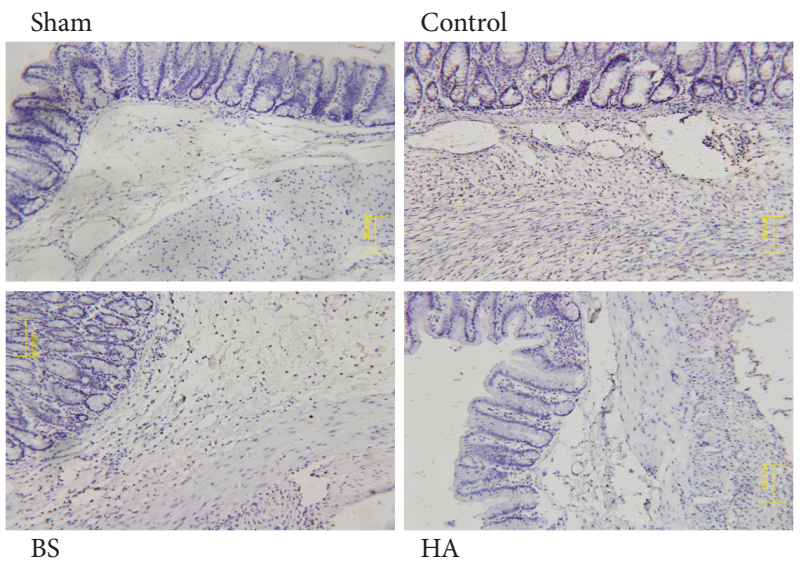

(a)

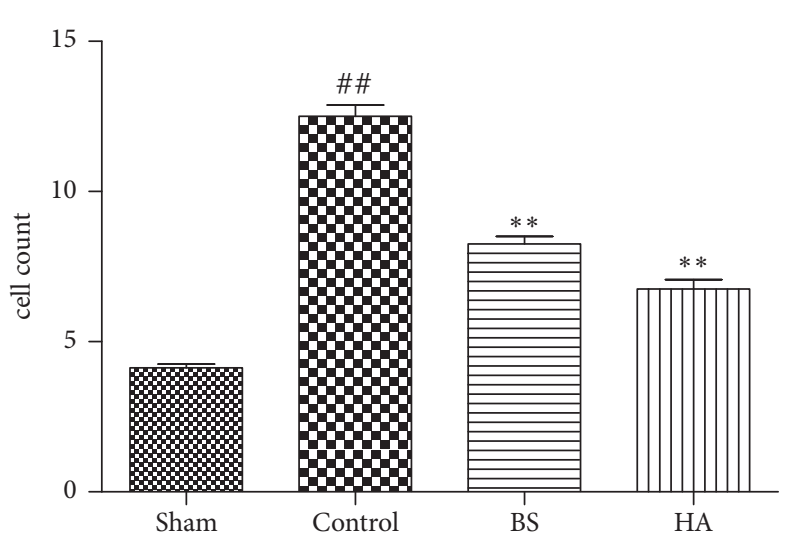

(b)

FIGURE 3: Bletilla striata reduces the TNF- $\alpha$ expression in caecum on the 7th day after operation. (a) The typical image of TNF- $\alpha$ expression among sham laparotomy, control, BS, and HA group by immunohistochemical staining. (b) Analysis of TNF- $\alpha$ cell count by immunohistochemical staining, $* * \mathrm{P}<0.01$ compared with the control group. \#\# $\mathrm{P}<0.01$ compared with the sham group.

no significant difference of IL- $1 \beta$ and TNF- $\alpha$ levels in rat's peritoneal fluid between the control group and the sham operation group, which is consistent with the findings of Chegini's research [19].

3.5. The Effect of Bletilla striata on Postoperative Intestinal Adhesion Is Closely Related to TGF- $\beta$ Signaling Pathway. We used TCMID to analyze the biopathway enrichment of the active ingredients of Bletilla striata in the Kyoto Encyclopedia of Genes and Genomes (KEGG) and found that mRNA surveillance pathway, TGF- $\beta$ signaling pathway, long-term depression, melanogenesis, and tyrosine metabolism were the enrichment pathways for Bletilla striata (Figure 5), so we confirm that TGF- $\beta$ signaling pathway is closely related to the efficacy of Bletilla striata.

3.6. Bletilla striata Inhibited the Levels of TGF- $\beta 1$ in the Cecum after PPAs in Rats. The concentration of TGF- $\beta 1$ in cecum tissue was detected by ELISA after PPAs in rats. The results showed that TGF- $\beta 1$ in the control group was significantly higher than that in the sham operation group $(\mathrm{P}<0.01)$. The secretion of TGF- $\beta 1$ in Bletilla striata group was significantly lower than that in control group $(\mathrm{P}<0.01)$. The secretion of TGF- $\beta 1$ in HA group was not significantly different from that in control group $(P>0.05)$ (Figure 6). These results show that Bletilla striata can prevent adhesion better than HA.

\subsection{Bletilla striata Reduced the Collagen Expression in Cecum}

Tissue. We examined the thickness of the collagen by Masson's trichrome stain. The blue stained collagen fibers could be detected within mucosal muscularis in sham operation group. In control group, the collagen fibers significantly increased around the mucosal muscularis and submucous layer. Bletilla striata and HA could significantly decrease the thickness of collagen fibers in the caecum of rats (Figure 7).
3.8. Bletilla striata Inhibited the Protein Expression of TGF$\beta 1$ and $\alpha-S M A$ in the Cecum Tissue after PPAs. We used the TCMID system to analyze TGF- $\beta$ signaling pathway and confirm that it is closely related to the preventing efficacy of Bletilla striata on PPAs, and so the key markers of epithelial-mesenchymal transition of $\alpha$-SMA and TGF- $\beta 1$ were determined by Western blotting. The results showed that $\alpha$-SMA and TGF- $\beta$ protein expression significantly increased in the rats of control group, and Bletilla striata and HA significantly decreased the protein expression of $\alpha$-SMA and TGF- $\beta(\mathrm{P}<0.01)$ (Figure 8$)$.

\section{Discussion}

The aim of this study was to evaluate the importance of Bletilla striata in preventing PPAs in rats. The current study found that Bletilla striata, a widely used astringent hemostatic medicinal plant, can effectively prevent PPAs by observing the degree of abdominal adhesion on the 7th day after operation. Hyaluronic acid (HA) is an anionic, nonsulfated glycosaminoglycan distributed in connective tissue [20-22]. Previous studies have pointed to the importance of HA in preventing the PPAs $[12,23]$. Therefore, in our study, the hyaluronic acid (HA) as a positive drug was used for the treatment of postoperative intestinal adhesion, and it was compared with Bletilla striata to observe their effect on the intestinal adhesion after operation.

Previous research has confirmed that inflammation of the entire peritoneum cavity is an important mechanism involved in adhesion formation [24]. Inflammatory reaction is one of the important pathophysiological processes leading to epithelial-mesenchymal transition [25]. Therefore we used the inflammation grading scale to compare inflammatory responses in four groups of rats [14]. The results showed that the inflammatory reaction in Bletilla striata group was significantly reduced compared with the control group, indicating 


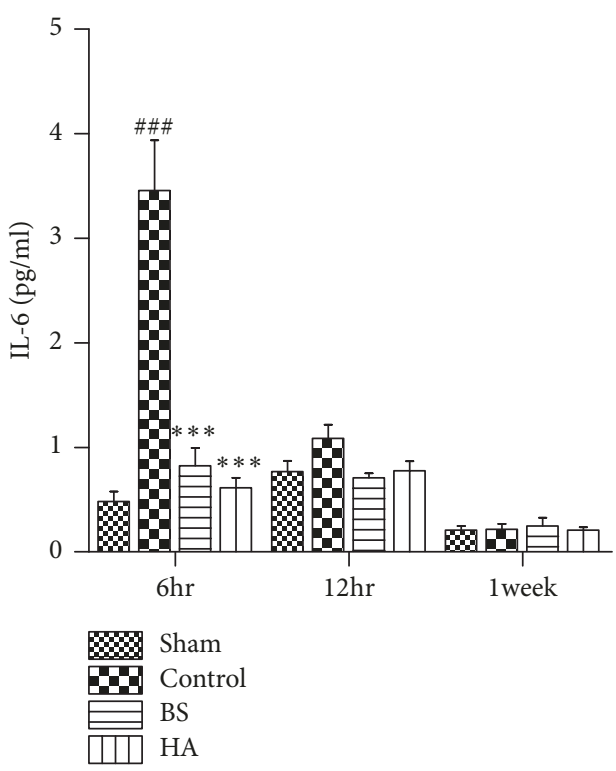

(a)

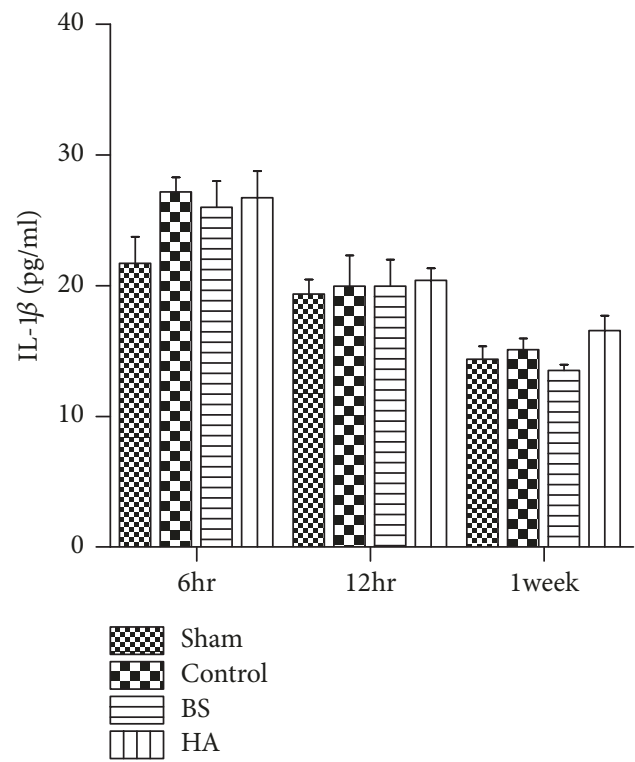

(c)

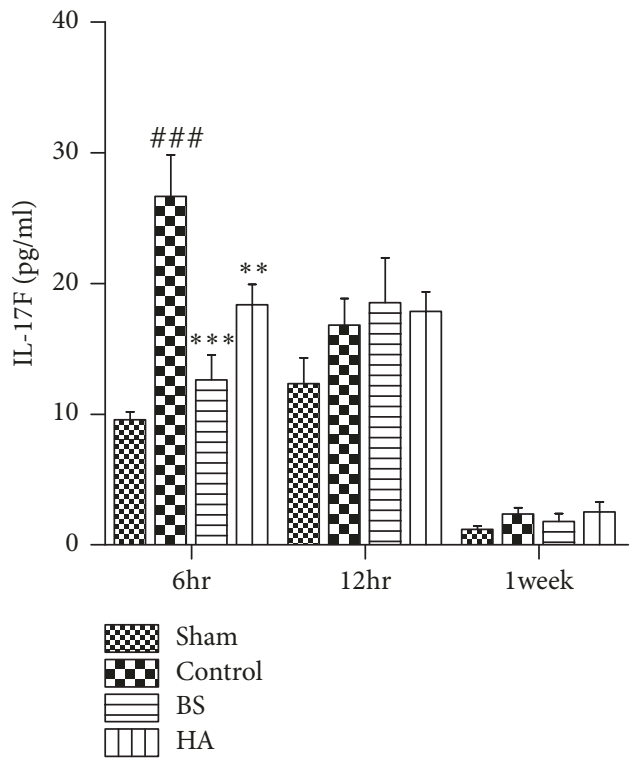

(b)

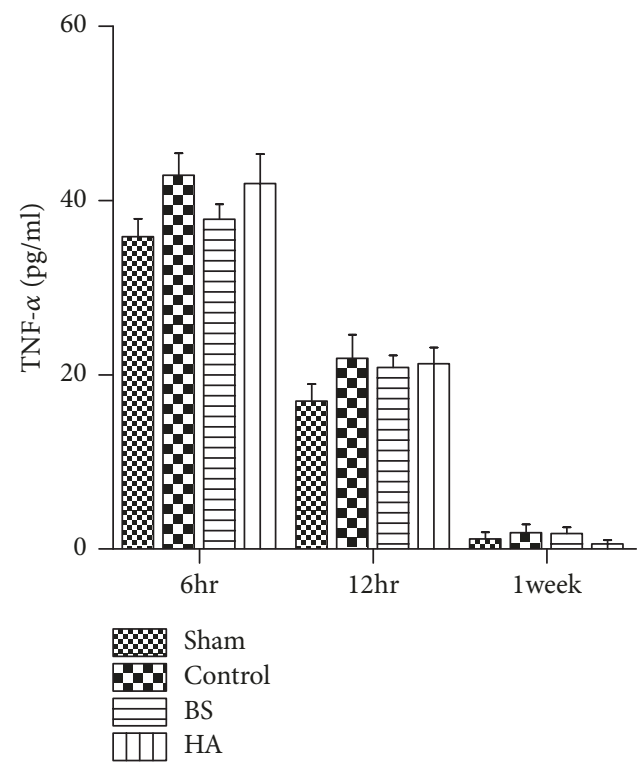

(d)

FIGURE 4: Bletilla striata inhibited the levels of IL-6 in the plasma and IL-17F in the peritoneal fluid after postoperative intestinal adhesion in rats. (a) Plasma IL-6, (b) IL-17F, (c) IL-1 $\beta$, and (d) TNF- $\alpha$ in peritoneal fluid levels were measured by ELISA assay at 6 h, 12 h, and 1 week after operation. $* * \mathrm{P}<0.01, * * * \mathrm{P}<0.001$ compared with the control group. \#\#\#P<0.001 compared with the sham group.

that Bletilla striata could obviously reduce the inflammatory reaction and prevent intestinal adhesion after operation.

In this experiment, we tested a variety of inflammatory factors to further study the inflammatory response. Concentrations of IL- 6 , IL- $1 \beta$, and TNF- $\alpha$ were changed in plasma and peritoneal fluid at various time points after incision [26]. In our study, concentrations of IL-6 in plasma and IL-17F, IL$1 \beta$, and TNF- $\alpha$ in peritoneal fluid were measured by ELISA assay at $6 \mathrm{~h}, 12 \mathrm{~h}$, and 1 week after operation. The results showed that there were significant differences between the groups only at 6 hours, and Bletilla striata and HA could inhibit the secretion of IL-6 in plasma at 6 hours and have no effect at $12 \mathrm{~h}$ and 1 week after operation. This result is consistent with that found by Fredriksson [26]. Surprisingly, the ELISA results of this study showed no significant difference in the concentration of IL- $1 \beta$ and TNF- $\alpha$ among the four groups at $6 \mathrm{~h}, 12 \mathrm{~h}$, and 1 week after operation, but this finding was consistent with that of Chegini [19]. Chegini explained that the celiac fluid content of the two cytokines was not related to the formation of peritoneal adhesion. Our study shows that IL- $1 \beta$ and TNF- $\alpha$ in peritoneal fluid cannot well reflect the severity of PPAs. Wang et al. have confirmed that 


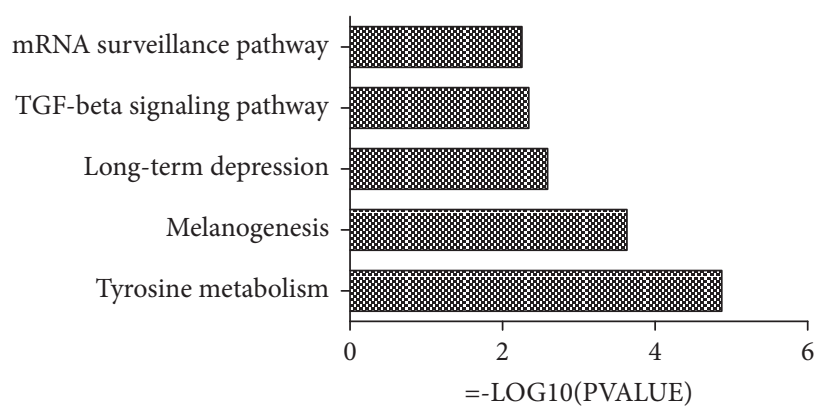

FIGURE 5: The effect of Bletilla striata on postoperative intestinal adhesion is closely related to TGF- $\beta$ signaling pathway. We used TCMID to analyze the biopathway enrichment of the active ingredients of Bletilla striata in the Kyoto Encyclopedia of Genes and Genomes (KEGG) and prove that TGF- $\beta$ signaling pathway was one of the most significant enrichment pathways for BS.

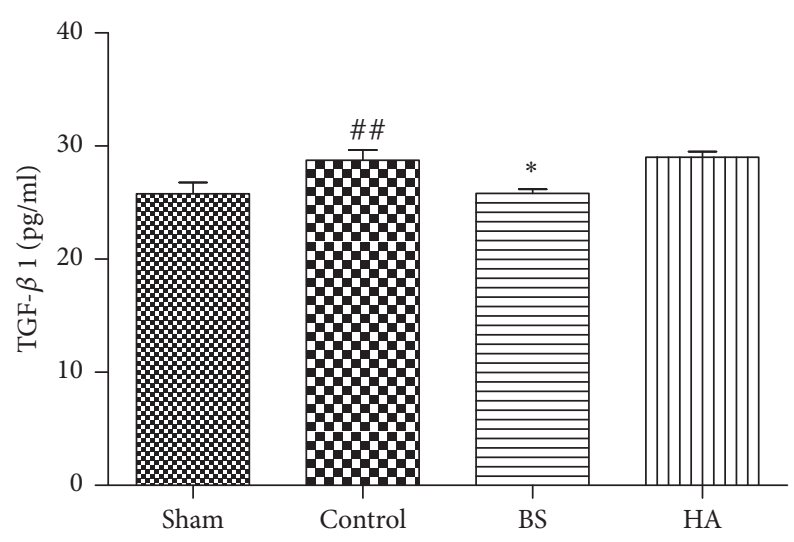

FIgURE 6: Bletilla striata inhibited the levels of TGF- $\beta 1$ in the cecum tissue after postoperative intestinal adhesion in rats. $* \mathrm{P}<0.05$ compared with the control group. \#\#P<0.05 compared with the sham group $(\mathrm{n}=8)$.

IL-17F enhances the response of fibroblasts to TGF- $\beta$ and is associated with adhesion formation [8]. The study of Chung found that IL-17F, a proinflammatory cytokine derived from $\mathrm{T}$ cells, showed a key role in adhesion formation [27]. Our study found that Bletilla striata could inhibit the secretion of IL-17F in peritoneal fluid at 6 hours and has no effect at $12 \mathrm{~h}$ and 1 week after operation.

We still conducted an immunohistochemistry experiment to test TNF- $\alpha$ level in cecum tissue because TNF- $\alpha$ has already been demonstrated as a good biological marker for PPAs, although TNF- $\alpha$ in peritoneal fluid is not a sensitive indicator [28]. Immunohistochemical results showed that TNF- $\alpha$ expressing cells were significantly greater in control group than in sham operation group. In Bletilla striata group, the expression of TNF- $\alpha$ was low in caecum. The inhibitory effect of Bletilla striata on PPAs was further proved.

We used the TCMID system to analyze TGF- $\beta$ signaling pathway and confirm that it is closely related to the preventing efficacy of Bletilla striata on PPAs. TGF- $\beta$, a substance that is expressed by all cell types, is thought to be a key factor in all stages of the wound healing process and is the major profibrotic cytokine. It can induce and be responsible for the activation of epithelial-mesenchymal transitions (EMTs). EMT is the process by which epithelial cells transform into mesenchymal cells and will eventually cause organ fibrosis $[25,29]$. TGF- $\beta$ will induce cell differentiation into myofibroblasts, which is highly associated with fibrotic diseases and will upregulate the expression of $\alpha$-SMA and collagens [30, 31]. The action of TGF- $\beta$ in tissue fibrosis appears to occur predominately through overexpression of TGF- $\beta 1$ compared with TGF- $\beta 2$ and TGF- $\beta 3$ isoforms [32]. Our study showed that Bletilla striata inhibited the levels of TGF- $\beta 1$ in the cecum tissue after postoperative intestinal adhesion in rats by ELISA and significantly decreased the thickness of collagen fibers in the caecum of rats by Masson's trichrome stain. Moreover Bletilla striata significantly decreased the protein expression of $\alpha$-SMA and TGF- $\beta$ by Western blotting. These results suggest that Bletilla striata could reduce the expression of the important substance which increased in PPAs.

The results of Figure 6 showed that HA could not significantly inhibit the secretion of TGF- $\beta 1$ compared with the control group, meaning that HA cannot completely prevent all of the pathological processes of adhesion. Wei et al. [33] have proved that HA could not inhibit the expression of TGF$\beta$ in peritoneal adhesion model of rats at the eighth day. Beyond that, a meta-analysis of four trials involving the use of hyaluronic acid [34-37] found no difference in overall mean adhesions scores [21]. A powder form containing hyaluronic acid was found to produce more adverse events [38]. These results further illustrate the importance of developing new drugs against surgical adhesion. Bletilla striata is an important astringent hemostatic medicine used for almost 2000 years in China and it has little side effect. At the same time, this study proved that Bletilla striata can effectively prevent the formation of adhesion after operation in rats, which is related to the inhibition of inflammatory factor IL-17F, IL6 , and TNF- $\alpha$ in different parts of rats, and can also prevent the expression of TGF- $\beta, \alpha$-SMA, and collagen fibers. All this indicates that Bletilla striata has potential antiadhesion effect and is worthy of further study to be developed into a clinical drug for antiadhesion after operation. 


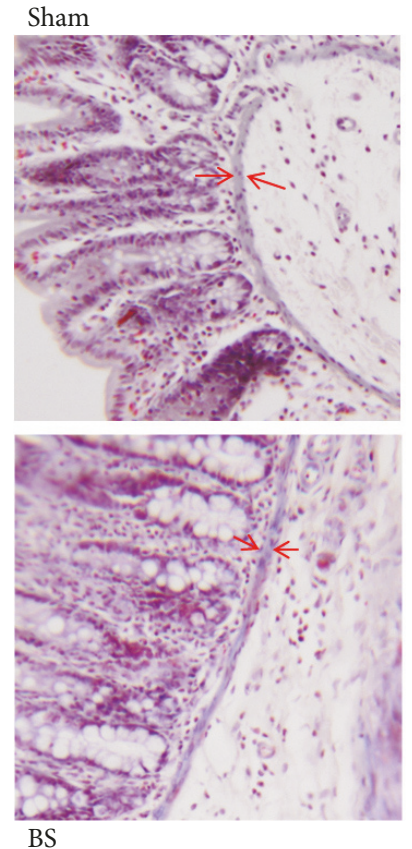

(a)
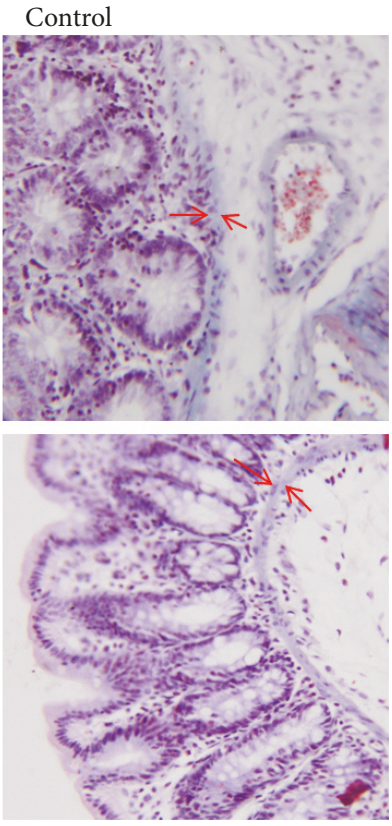

HA

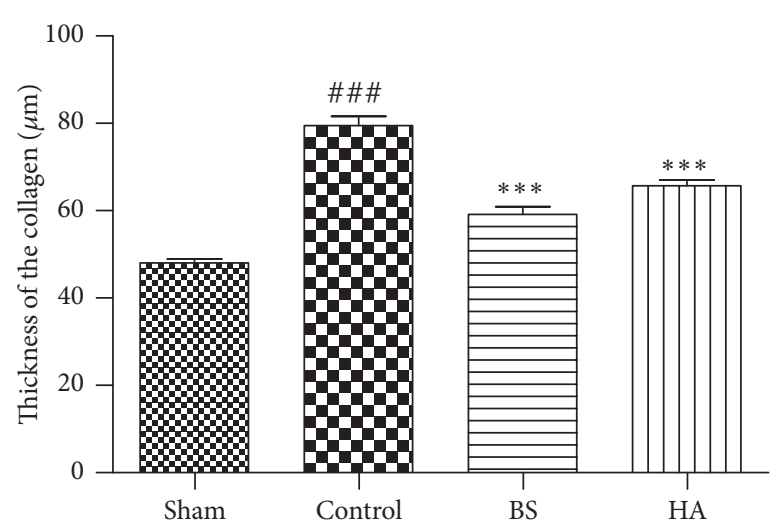

(b)

Figure 7: Bletilla striata reduced the collagen expression in cecum tissue on the 7th day after operation. (a) The typical image of collagen expression among sham laparotomy, control, BS, and HA group by Masson's trichrome stain. (b) Analysis of collagen thickness by Masson's trichrome stain. $* * * \mathrm{P}<0.01$ compared with the control group. \#\#\# $<0.001$ compared with the sham group.

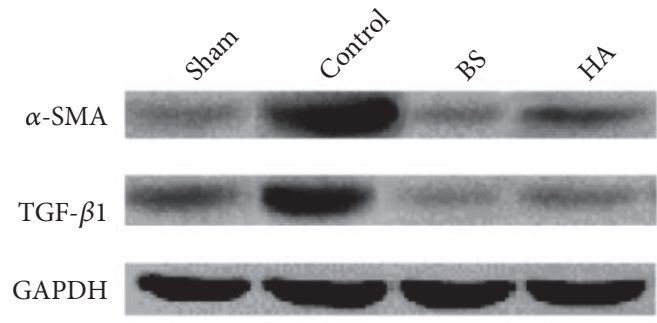

(a)

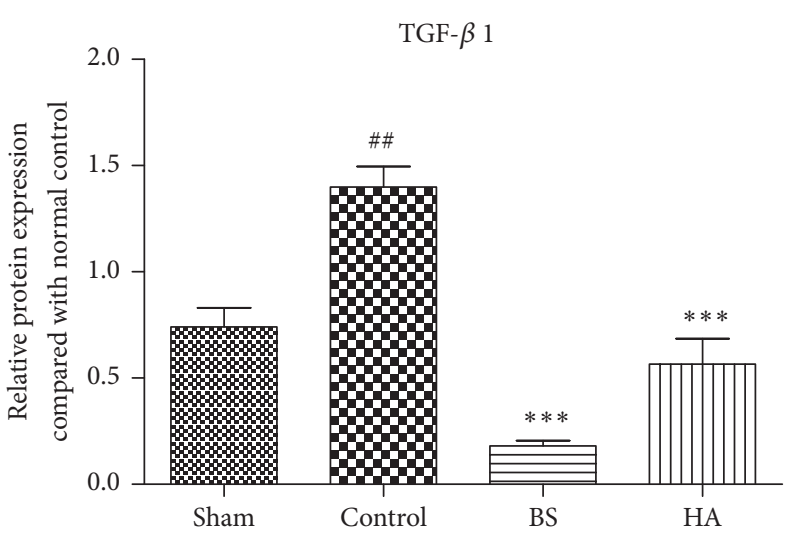

(b)

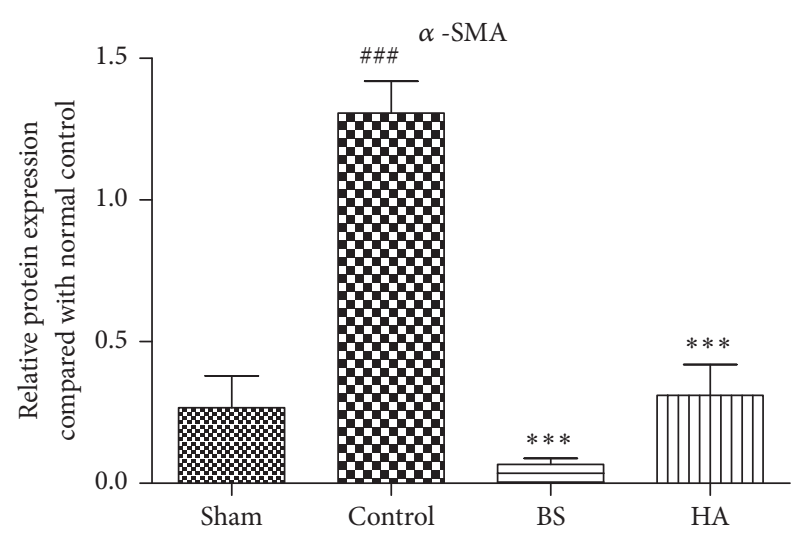

(c)

FIGURE 8: Bletilla striata inhibited the protein expression of TGF- $\beta 1$ and $\alpha$-SMA in the cecum tissue after postoperative intestinal adhesion in rats. (a) TGF- $\beta 1$ and $\alpha$-SMA protein bands of Western blot; (b) TGF- $\beta 1$ protein expression; (c) $\alpha$-SMA protein expression. $* * * \mathrm{P}<0.001$ compared with the control group. \#\#P<0.01, \#\#\# $\mathrm{P}<0.001$ compared with the sham group $(\mathrm{n}=3)$. 


\section{Data Availability}

The data used to support the findings of this study are available from the corresponding author upon request.

\section{Conflicts of Interest}

The authors have no conflicts of interest to declare.

\section{References}

[1] K. Okabayashi, H. Ashrafian, E. Zacharakis et al., "Adhesions after abdominal surgery: a systematic review of the incidence, distribution and severity," Surgery Today, vol. 44, no. 3, pp. 405420, 2014

[2] M. P. Diamond and M. L. Freeman, "Clinical implications of postsurgical adhesions," Human Reproduction Update, vol. 7, no. 6, pp. 567-576, 2001.

[3] R. P. G. Ten Broek, M. W. J. Stommel, C. Strik, C. J. H. M. Van Laarhoven, F. Keus, and H. Van Goor, "Benefits and harms of adhesion barriers for abdominal surgery: a systematic review and meta-analysis," The Lancet, vol. 383, no. 9911, pp. 48-59, 2014.

[4] H. Ellis, "Medicolegal consequences of postoperative intraabdominal adhesions," Journal of the Royal Society of Medicine, vol. 94, no. 7, pp. 331-332, 2001.

[5] B. W. J. Hellebrekers and T. Kooistra, "Pathogenesis of postoperative adhesion formation," British Journal of Surgery, vol. 98, no. 11, pp. 1503-1516, 2011.

[6] P. R. Koninckx, V. Gomel, A. Ussia, and L. Adamyan, "Role of the peritoneal cavity in the prevention of postoperative adhesions, pain, and fatigue," Fertility and Sterility, vol. 106, no. 5, pp. 998-1010, 2016.

[7] X. He, X. Wang, J. Fang et al., "Bletilla striata: medicinal uses, phytochemistry and pharmacological activities," Journal of Ethnopharmacology, vol. 195, pp. 20-38, 2017.

[8] Y. Wang, D. Liu, S. Chen, Y. Wang, H. Jiang, and H. Yin, "A new glucomannan from Bletilla striata: Structural and anti-fibrosis effects," Fitoterapia, vol. 92, pp. 72-78, 2014.

[9] G. S. diZerega and J. D. Campeau, "Peritoneal repair and postsurgical adhesion formation," Human Reproduction Update, vol. 7, no. 6, pp. 547-555, 2001.

[10] J.-J. Duron, "Postoperative intraperitoneal adhesion pathophysiology," Colorectal Disease, vol. 9, no. s2, pp. 14-24, 2007.

[11] O. Hemadeh, S. Chilukuri, V. Bonet, S. Hussein, and I. H. Chaudry, "Prevention of peritoneal adhesions by administration of sodium carboxymethyl cellulose and oral vitamin E," Surgery, vol. 114, no. 5, pp. 907-910, 1993.

[12] M. M. P. J. Reijnen, E. M. Skrabut, V. A. Postma, J. W. Burns, and H. Van Goor, "Polyanionic polysaccharides reduce intraabdominal adhesion and abscess formation in a rat peritonitis model," Journal of Surgical Research, vol. 101, no. 2, pp. 248-253, 2001.

[13] S. K. Nair, I. K. Bhat, and A. L. Aurora, "Role of proteolytic enzyme in the prevention of postoperative intraperitoneal adhesions," JAMA Surgery, vol. 108, no. 6, pp. 849-853, 1974.

[14] G. D. Hooker, B. M. Taylor, and D. K. Driman, "Prevention of adhesion formation with use of sodium hyaluronate-based bioresorbable membrane in a rat model of ventral hernia repair with polypropylene mesh - a randomized, controlled study," Surgery, vol. 125, no. 2, pp. 211-216, 1999.
[15] M. Park, S. Moon, E. Lee et al., "IL-1-IL-17 signaling axis contributes to fibrosis and inflammation in two different murine models of systemic sclerosis," Frontiers in Immunology, vol. 9, article 1611, 2018.

[16] H. Guo, J. C. K. Leung, M. F. Lam et al., "Smad7 transgene attenuates peritoneal fibrosis in uremic rats treated with peritoneal dialysis," Journal of the American Society of Nephrology, vol. 18, no. 10, pp. 2689-2703, 2007.

[17] R. Xue, Z. Fang, M. Zhang, Z. Yi, C. Wen, and T. Shi, “TCMID: Traditional Chinese medicine integrative database for herb molecular mechanism analysis," Nucleic Acids Research, vol. 41, no. 1, pp. D1089-D1095, 2013.

[18] Z. Liu, F. Guo, Y. Wang et al., "BATMAN-TCM: a bioinformatics analysis tool for molecular mechanism of traditional chinese medicine," Scientific Reports, vol. 6, article 21146, 2016.

[19] N. Chegini, H. Rong, B. Bennett, and I. K. Stone, "Peritoneal fluid cytokine and eicosanoid levels and their relation to the incidence of peritoneal adhesion," Journal of the Society for Gynecologic Investigation, vol. 6, no. 3, pp. 153-157, 1999.

[20] C.-L. Tang, D. G. Jayne, F. Seow-Choen, Y.-Y. Ng, K.-W. Eu, and N. Mustapha, "A randomized controlled trial of $0.5 \%$ ferric hyaluronate gel (intergel) in the prevention of adhesions following abdominal surgery," Annals of Surgery, vol. 243, no. 4, pp. 449-455, 2006.

[21] M. Metwally, D. Gorvy, A. Watson, and T. C. Li, "Hyaluronic acid fluid agents for the prevention of adhesions after fertilitypreserving gynecological surgery: a meta-analysis of randomized controlled trials," Fertility and Sterility, vol. 87, no. 5, pp. 1139-1146, 2007.

[22] V. Mais, M. G. Cirronis, M. Peiretti, G. Ferrucci, E. Cossu, and G. B. Melis, "Efficacy of auto-crosslinked hyaluronan gel for adhesion prevention in laparoscopy and hysteroscopy: a systematic review and meta-analysis of randomized controlled trials," European Journal of Obstetrics \& Gynecology and Reproductive Biology, vol. 160, no. 1, pp. 1-5, 2012.

[23] G. Ahmad, H. O’Flynn, A. Hindocha, and A. Watson, "Barrier agents for adhesion prevention after gynaecological surgery," Cochrane Database of Systematic Reviews, vol. 4, Article ID CD000475, 2015.

[24] R. Corona, J. Verguts, R. Schonman, M. M. Binda, K. Mailova, and P. R. Koninckx, "Postoperative inflammation in the abdominal cavity increases adhesion formation in a laparoscopic mouse model," Fertility and Sterility, vol. 95, no. 4, pp. 1224$1228,2011$.

[25] R. Kalluri and R. A. Weinberg, "The basics of epithelialmesenchymal transition," The Journal of Clinical Investigation, vol. 119, no. 6, pp. 1420-1428, 2009.

[26] F. Fredriksson, R. H. Christofferson, P. O. Carlsson, and H. E. Lilja, "Locally increased concentrations of inflammatory cytokines in an experimental intraabdominal adhesion model," Journal of Pediatric Surgery, vol. 49, no. 10, pp. 1480-1484, 2014.

[27] D. R. Chung, T. Chitnis, R. J. Panzo, D. L. Kasper, M. H. Sayegh, and A. O. Tzianabos, "CD4+ T cells regulate surgical and postinfectious adhesion formation," The Journal of Experimental Medicine, vol. 195, no. 11, pp. 1471-1478, 2002.

[28] A. A. Kaidi, T. Gurchumelidze, M. Nazzal, P. Figert, C. Vanterpool, and Y. Van Silva, "Tumor necrosis factor- $\alpha$ : A marker for peritoneal adhesion formation," Journal of Surgical Research, vol. 58, no. 5, pp. 516-518, 1995.

[29] R. Kalluri and E. G. Neilson, "Epithelial-mesenchymal transition and its implications for fibrosis," The Journal of Clinical Investigation, vol. 112, no. 12, pp. 1776-1784, 2003. 
[30] A. Biernacka, M. Dobaczewski, and N. G. Frangogiannis, "TGF$\beta$ signaling in fibrosis," Growth Factors, vol. 29, no. 5, pp. 196202, 2011.

[31] E. Pardali, G. Sanchez-Duffhues, M. Gomez-Puerto, and P. ten Dijke, “TGF- $\beta$-induced endothelial-mesenchymal transition in fibrotic diseases," International Journal of Molecular Sciences, vol. 18, no. 10, article 2157, 2017.

[32] N. Chegini, “TGF- $\beta$ system: the principal profibrotic mediator of peritoneal adhesion formation," Seminars in Reproductive Medicine, vol. 26, no. 4, pp. 298-312, 2008.

[33] G. Wei, X. Chen, G. Wang, L. Fan, K. Wang, and X. Li, "Effect of resveratrol on the prevention of intra-abdominal adhesion formation in a rat model," Cellular Physiology and Biochemistry, vol. 39, no. 1, pp. 33-46, 2016.

[34] M. P. Diamond, K. Bachus, E. Bieber et al., "Reduction of de novo postsurgical adhesions by intraoperative precoating with sepracoat (HAL-C) solution: a prospective, randomized, blinded, placebo-controlled multicenter study," Fertility and Sterility, vol. 69, no. 6, pp. 1067-1074, 1998.

[35] D. B. Johns, G. M. Keyport, F. Hoehler, and G. S. DiZerega, "Reduction of postsurgical adhesions with intergel adhesion prevention solution: a multicenter study of safety and efficacy after conservative gynecologic surgery," Fertility and Sterility, vol. 76, no. 3, pp. 595-604, 2001.

[36] P. Lundorff, H. Van Geldorp, S. E. Tronstad et al., "Reduction of post-surgical adhesions with ferric hyaluronate gel: a European study," Human Reproduction, vol. 16, no. 9, pp. 1982-1988, 2001.

[37] M. Pellicano, S. Bramante, D. Cirillo et al., "Effectiveness of autocrosslinked hyaluronic acid gel after laparoscopic myomectomy in infertile patients: a prospective, randomized, controlled study," Fertility and Sterility, vol. 80, no. 2, pp. 441-444, 2003.

[38] S. V. Berdah, C. Mariette, C. Denet et al., "A multicentre, randomised, controlled trial to assess the safety, ease of use, and reliability of hyaluronic acid/carboxymethylcellulose powder adhesion barrier versus no barrier in colorectal laparoscopic surgery," Trials, vol. 15, no. 1, article 413, 2014. 


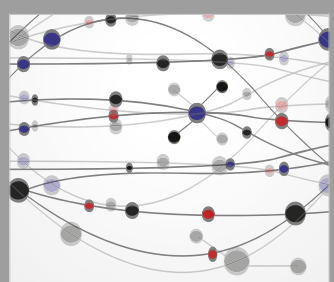

The Scientific World Journal
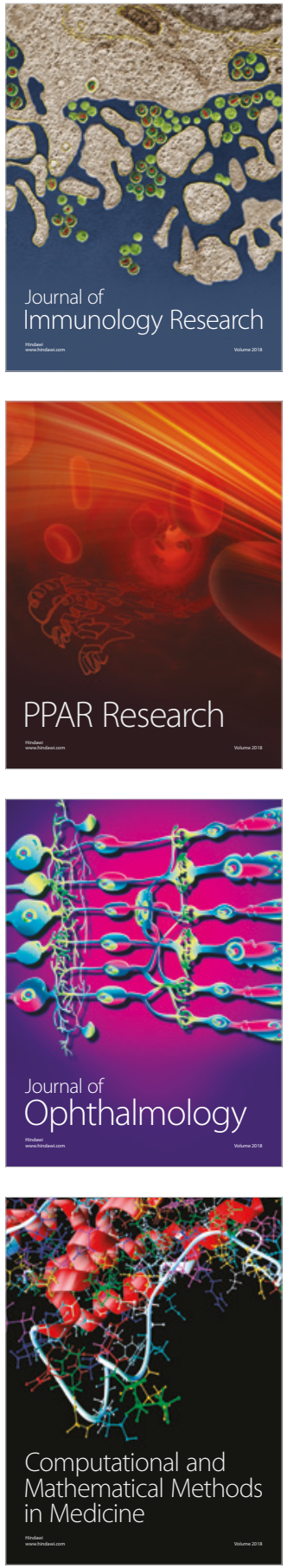

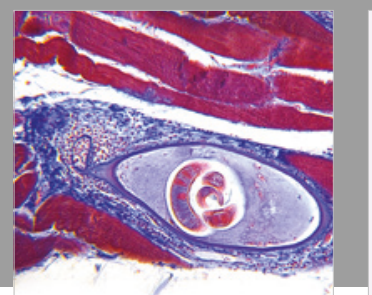

Gastroenterology Research and Practice

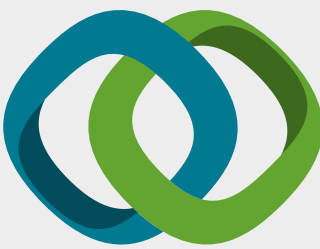

\section{Hindawi}

Submit your manuscripts at

www.hindawi.com
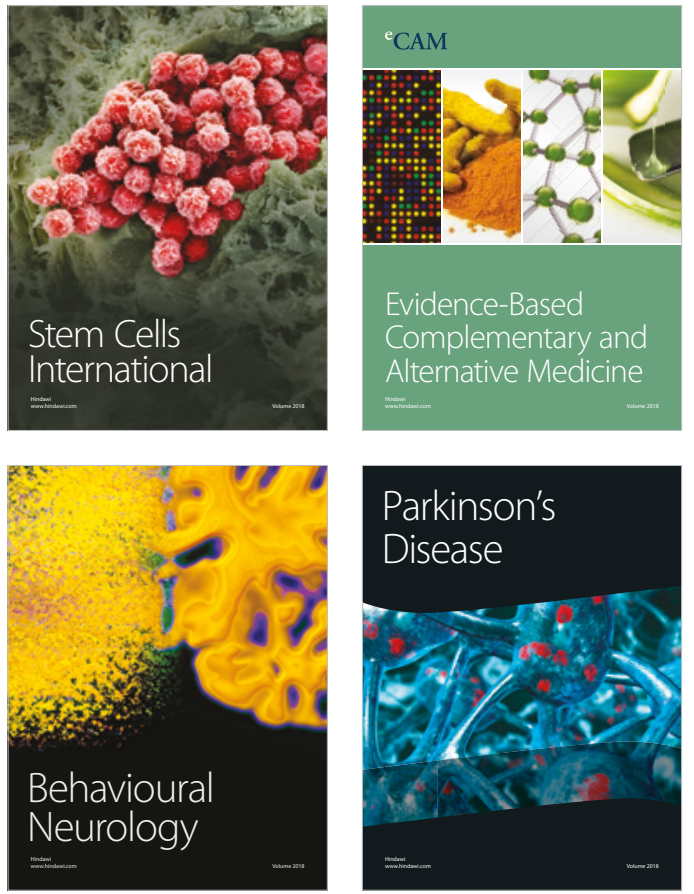

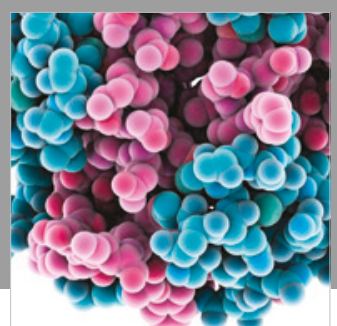

ournal of

Diabetes Research

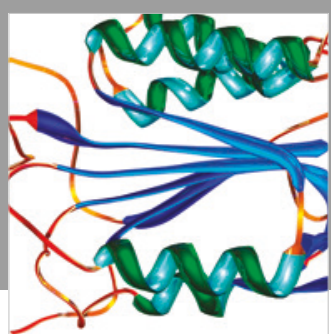

Disease Markers
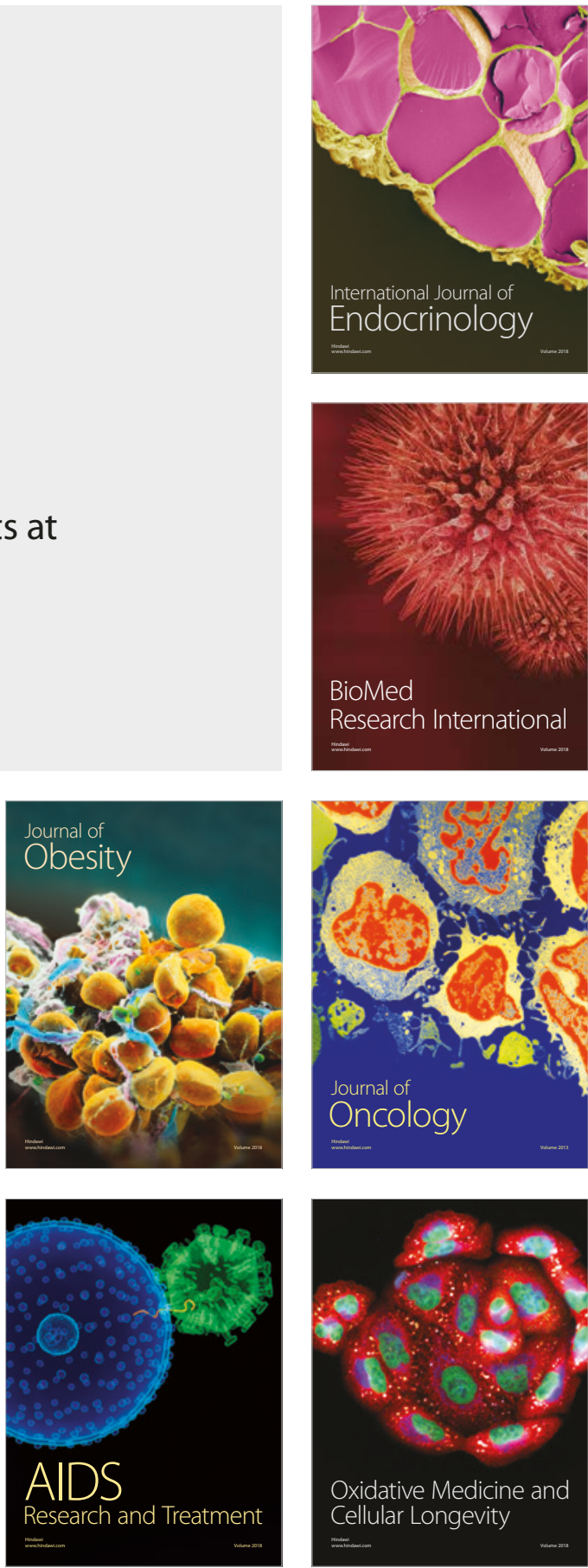